\title{
Combined Procedure of Aspiration Termination and Laparoscopic Sterilization
}

\author{
P. C. STEPTOE,* F.R.C.S.ED., F.R.C.o.G. ; M. IMRAN, $†$ M.B., B.S.
}

\begin{abstract}
Summary : Termination of pregnancy and laparoscopic $N$ sterilization has been performed in 101 women in a one-year period. The complication rate was low and the average stay in hospital was short. With close supervision and co-operation between the gynaecologist and the patient's general practitioner it is suggested that this is a safe and economic procedure.
\end{abstract}

\section{Introduction}

Termination of pregnancy by aspiration technique has become established as one of the safest methods of therapeutic abortion up to about the sixteenth week. The optimum time to carry out this procedure is at eight weeks, since the cervix does not then need more than a No. 12 Hegar dilator and the risk of accidental perforation of the uterus is small. Further, at this stage of pregnancy the aspiration is carried out rapidly, and the products of conception are removed readily and completely, with loss of less than $300 \mathrm{ml}$. of blood. At 12 weeks dilatation and introduction of a large aspiration cannula of No. 14 Hegar size is necessary, and the conception material is broken up and sucked out piecemeal. If the uterus is larger than this, aspiration has to be accompanied by exploration of the uterine cavity with ring or ovum forceps. The operation is then prolonged, and there is much heavier bleeding, which may require immediate replacement of the amount of blood lost. Incomplete emptying of the uterus is a real risk, which could lead to infection, persistent bleeding, and the necessity of a second operation. The cervix may be seriously damaged by the degree of dilatation necessary, with the subsequent development of chronic cervicitis, often accompanied by incompetence of the internal cervical os. These sequelae are obviously undesirable in all patients, but particularly so in the young nullipara.

The indications for terminating pregnancy are by no means the same as those for sterilization of the woman. Nevertheless, there are many patients of high parity whose pregnancy is to be terminated in whom the risk of further pregnancy is clearly not desired or desirable. The majority of these patients will be over 30 years of age, and many will have no symptoms or history of previous gynaecological disorders. This point is important, because previous menorrhagia or prolapse often predetermines the method of sterilization to be offered, which may even include hysterectomy. In the former group of patients with a healthy pelvis sterilization by laparoscopic technique at the time of evacuation of the uterus is a very attractive proposition, which avoids formal invasion of the abdominal cavity with its relatively greater disturbance and long hospital stay. In the past such patients were usually treated by abdominal hysterotomy and tubal sterilization, by means of the Madlener or Pomeroy techniques, or even excision of the tubes. Sterilization by laparoscopic technique adds only five minutes to the operation of aspiration by the vaginal route, and does not prolong the stay in hospital. Indeed, in our unit we have carried out this double procedure on selected cases as day patients. The women stay in for about nine hours, and are then dis-

* Consultant Obstetrician and Gynaecologist, Oldham Hospital Group. t Registrar, Obstetrical and Gynaecological Department, Oldham and
District General Hospital. charged home to the care of their general practitioner-a very important person in the successful management of the case. Preoperative assessment of these patients must exclude any complicating medical condition, infection, or anaemia which would not permit such a rapid progress through the hospital.

\section{Technique}

Evacuation of the Uterus.-Under general anaesthesia the uterus is evacuated by the technique described by Vojta (1967), with the following modifications. A modified lithotomy position is used and an intravenous injection of 1 ampoule of Syntometrine is given at the onset of cervical dilatation. In primigravidae dilatation of the cervix may be facilitated by paracervical injection of $1 \%$ lignocaine solution. At the conclusion of the evacuation the large uterine cannula is replaced by a small one, and this is used together with a vulsellum forceps to manipulate the uterus.

Sterilization.-Sterilization by diathermy and division of the isthmus of each tube is now carried out by the technique described by Steptoe $(1967,1969)$. The essential features are an adequate pneumoperitoneum induced with carbon dioxide, fibreoptic laparoscope, and a partial Trendelenburg position. The technique requires especial care in securing the tubes with the biopsy forceps drill. This is because the uterus after its evacuation occupies most of the pelvis and the tubes are displaced outwards and may well be lying over the brim of the pelvis. The tissues beneath the peritoneum here are in danger if the forceps are carelessly applied. Attention to detail avoids picking up or damaging any tissue except the tubal targets. During the procedure care is taken to ensure that the operation area is not invaded by any other organ such as bowel. The laparoscope, of course, allows one to make a detailed examination of the uterus to assess its contraction and to detect any evidence of uterine perforation. At the conclusion of the sterilization and on closure of the two small incisions a bimanual examination and massage assures good retraction of the uterus after removal of the cervical instruments. Intramuscular ergometrine may be prescribed during the ensuing few hours of recovery. Any blood loss in excess of $500 \mathrm{ml}$. should be replaced while the patient is still unconscious either by blood or by dextran solution, utilizing the convenient polyethylene cannula used by the anaesthetist for medication during the operation.

\section{Discussion}

The accompanying Table shows the number of patients and the methods of termination used in our unit during the first 12 months following inception of the new Abortion Act.

\section{Legal Abortions 1968-9}

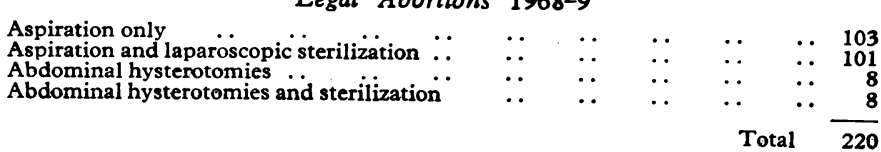


The mean duration of stay in hospital for patients undergoing abdominal hysterotomy was $10 \cdot 1$ days. The average stay after aspiration and laparoscopic sterilization was $2 \cdot 4$ days, the shortest being nine hours. In this latter group the mean age of the patients was 32.6 years and the average number of children 3.5.

Complications occurred in only seven cases of the series, and there were no maternal deaths. The details of these complications are as follows:

Three cases of chronic nephritis and one case of mitral valve disease developed mild pyrexia, and were treated with penicillin.

One case of incomplete aspiration of the uterus needed a repeat evacuation of retained products, blood transfusion, and antibiotics.

One case required readmission because of a pelvic abscess; this was drained through a posterior colpotomy incision.

One case had damage to vessels of the mesosalpinx which was not readily controlled, and laparotomy was immediately performed with suture of the damaged vessels.

As a rule antibiotics were not prescribed without first taking swabs from the cervix and vagina for bacteriological examination, identification, and sensitivity. In the pyrexial cases the urine was also examined for possible infection as a routine. Blood grouping and haemoglobin estimations were also carried out in all cases. The amount of blood loss was always measured. Blood transfusion was used in only two patients in whom the preoperative haemoglobin level was below $73 \%$.

The criteria for choosing to carry out the procedures on a day-patient basis were the absence of a complicating medical disease or signs of pelvic infection, the presence of a preoperative haemoglobin of $80 \%$, and no occurrence of any complication during the operation. The procedure should be carried out only where full hospital facilities are available, and where preparation for a laparotomy has previously been made. The day cases received atropine $0.6 \mathrm{mg}$. only half an hour before operation. A short-acting general anaesthetic was always used, and full resuscitation facilities and equipment were available in a large recovery bay next to the theatre which could accommodate six patients. Intramuscular ergometrine $0.5 \mathrm{mg}$. was given half an hour before discharge to the patient's home. The patient's general practitioner was informed of the proposed plan of discharge from the hospital, and was also contacted during the morning of the operation on the day patients.

In these circumstances it is believed that this combined procedure has a definite place in the gynaecological repertory, being safe, economic in usage of beds, and without disturbance of patients (Steptoe, 1969).

\section{REFERENCES}

Steptoe, P. C. (1967). Laparoscopy in Gynaecology. Edinburgh, Livingstone.

Steptoe, P. C. (1969). Laparoscopic Sterilization. Report of 500 cases. Proceedings of Symposium at the Royal College of Obstetricians and Gynaecologists. In press.

Vojta, M. (1967). Fournal of Obstetrics and Gynaecology of the British Commonwealth, 74, 768 .

\title{
Third Case of Chronic Lymphocytic Leukaemia in a Carrier of the Inherited $\mathrm{Ch}^{1}$ Chromosome
}

\author{
P. H. FITZGERALD,* PH.D. ; J. W. HAMER, $\dagger$ M.B., M.R.A.C.P., M.C.PATH.
}

Summary : Chronic lymphocytic leukaemia has devel$S$ oped in three siblings who are all carriers of the abnormal $\mathrm{Ch}^{1}$ chromosome. ${ }^{3} \mathrm{H}$-thymidine autoradiography showed $\mathrm{Ch}^{1}$ to be a $\mathbf{G}_{2}$ autosome, from which the short arm is absent, and not $G_{1}$, which is trisomic in Down's syndrome and which is believed to form the abnormal $\mathrm{Ph}^{1}$ chromosome. $\mathrm{Ch}^{1}$ is not of general significance in the aetiology of chronic lymphocytic leukaemia, but it must be considered as a possible predisposing factor to leukaemia in the present family.

\section{Introduction}

An inherited chromosomal abnormality $\left(\mathrm{Ch}^{1}\right)$ carried by several members of a family, including two who had developed chronic lymphocytic leukaemia, was reported by Gunz et al. (1962). The abnormal chromosome was a $G$ group autosome which had lost all or the greater part of its short arm. We now

* Director, Cytogenetics Unit, Christchurch Hospital, Christchurch, New

†ealand. Zealand.

The Cytogenetics Unit of Christchurch Hospital is supported by the Canterbury and Westland Division of the Cancer Society of New Zealand. report the development of chronic lymphocytic leukaemia in a third sibling and carrier of the abnormal chromosome.

The revised pedigree of the $\mathrm{C}$. family, with further information on the inheritance of $\mathrm{Ch}^{1}$, is shown in Fig. 1. Nos. 3, 4, and 6, all carriers of $\mathrm{Ch}^{1}$, now have chronic lymphocytic leukaemia.

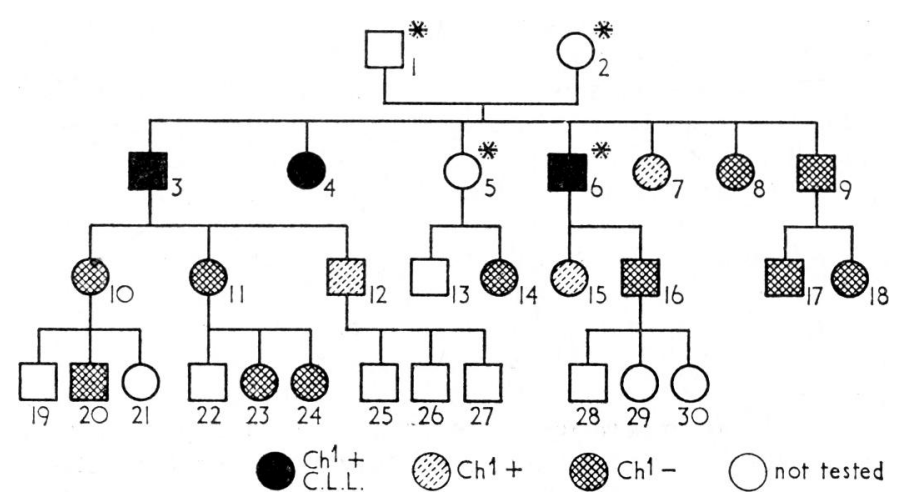

FIg. 1.-Pedigree of the C. family. $\mathrm{Ch}^{2}+=\mathrm{Ch}^{2}$ chromosome present. $\mathrm{Ch}^{1}-\mathrm{Ch}^{1}$ chromosome not present. C.L.L.=Chronic lymphocytic leukaemia. * Deceased. 